\title{
Sex hormones, autoimmunity and gender disparity in COVID-19
}

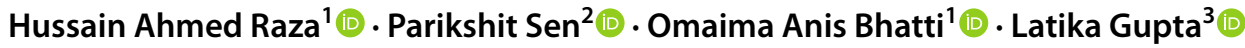

Received: 21 February 2021 / Accepted: 19 April 2021 / Published online: 26 April 2021

(c) The Author(s), under exclusive licence to Springer-Verlag GmbH Germany, part of Springer Nature 2021

\begin{abstract}
The Coronavirus disease 2019 (COVID-19) pandemic has majorly contributed to massive and widespread mortality. Epidemiological data strongly indicates a sex-based disparity in COVID-19 clinical outcomes, with women having lower infection and hospitalisation rates, coupled with better prognosis and lesser mortality. This disparity may be explained by several mechanisms including differences in innate and adaptive immune responses, genetic factors, and an interplay between sex hormones and immune effectors, as well as gender-specific behaviour differences. These pathways, particularly the immunological divergence in response to viral infection, could potentially influence not only COVID-19 pathogenesis and disease course, but also the response to antiviral drugs and vaccines. Furthermore, factors that confer a protective advantage against COVID-19 may be exploited to develop therapeutic strategies to improve clinical outcomes in COVID-19.
\end{abstract}

Keywords COVID-19 · Coronavirus · Gender bias $\cdot$ Sex differences $\cdot$ Clinical outcomes

\section{Introduction}

The Coronavirus disease 2019 (COVID-19) pandemic continues to be a significant cause of morbidity and mortality worldwide in the second year following its inception. Initial demographic accounts from Wuhan, China that indicated a higher mortality in men as compared to women, were further corroborated by numerous epidemiological studies that reported sex-based disparities in disease severity and clinical outcomes of COVID-19 [1].

Latika Gupta

drlatikagupta@gmail.com

Hussain Ahmed Raza

hussain_gorz@outook.com

Parikshit Sen

parikshit.sen@hotmail.com

Omaima Anis Bhatti

omaimaanisbhatti@gmail.com

1 Medical College, Aga Khan University Hospital, Stadium Road, Karachi, Pakistan

2 Maulana Azad Medical College, 2-Bahadur Shah Zafar marg, New Delhi, India

3 Department of Clinical Immunology and Rheumatology, Sanjay Gandhi Postgraduate Institute of Medical Sciences, Lucknow 226014, India
The Chinese Centre for Disease Control and prevention reported the ratio of male to female COVID-19 infections as 2.7:1, and a Case Fatality Rate (CFR) of $2.8 \%$ in males as compared to $1.7 \%$ in females, calculated from a group of 72 , 314 patients in China [2,3]. This was further supported by the Global Health 50/50 sex-disaggregated data, which indicated 14 male deaths for every 10 female deaths resulting from COVID-19. Epidemiological studies from 38 countries reported a mean CFR in males that was 1.7 times higher than the mean CFR in females, with advancing age being a risk factor for mortality in both sexes [4, 5]. European studies reported greater case fatality rates in males above 20 years, with a cohort study involving 17 million adults in England indicating a significant correlation between male sex and risk of COVID-19-associated mortality, with a hazard ratio of 1.59 [4]. In conclusion, current epidemiological evidence suggests a bias towards males regarding greater susceptibility to both SARS-CoV-2 infection as well as to severe COVID-19 clinical outcomes. While this is concerning, it is unsurprising as similar sex-based disparities were previously also observed in the Severe Acute Respiratory (SARS) and Middle East Respiratory Syndrome (MERS) epidemics [6, 7]. The European League against Rheumatism (EULAR) COVID-19 registry which collected data on COVID-19 in people with rheumatic diseases (RDs) stands out as an exception, with 4018 (65\%) of a total 6155 patients with COVID-19 being female. However, since an increased 
incidence of RDs is well-documented in women, this could plausibly bias the sample population, leading to greater COVID-19 being observed in women with RDs, as opposed to a sample from the general population [8]. Furthermore, as per the data from this study, being a male over 65 was identified as the single most important risk factor COVID-19 hospitalisation in people with inflammatory rheumatic and musculoskeletal diseases (RMDs) [9].

A physiological basis for an inherent protective effect against COVID-19 disease severity in females may potentially be exploited to develop a therapeutic intervention for better management of COVID-19. This review investigates the sex-based differences in genetics, behaviour, hormones, and the immune response to suggest a plausible explanation for the gender-based disparity evident in COVID-19 clinical outcomes and mortality [10].

\section{Search strategy}

A literature review was conducted as per the review recommendations by Gasparyan et al. [11]. A search was conducted using PubMed, MEDLINE, EMBASE, ClinicalTrials.gov and SCOPUS databases, using the terms "sex differences OR sex disparity OR gender differences OR gender bias" AND "COVID-19 OR SARS-CoV2 OR SARSCOV-2 OR novel coronavirus OR nCOV". Approximately 1500 articles were identified, and ranged from case reports, case series, and letters to the editor, and observational studies to narrative reviews, systematic reviews and clinical trials published till March 26, 2021. Relevant articles were independently reviewed, and information was extracted falling under the different categories of; sex differences in immune response, role of sex hormones in immune response, gender differences in COVID-19 infections and outcomes, COVID19 and thrombosis, COVID-19 and pregnancy, COVID-19 and autoimmunity and gender differences in the vaccine response.

\section{Gender-specific differences in immune response}

Women are known to inherently mount a stronger innate and adaptive immune response to viral infection. Faster viral clearance and a lower viral load have also been reported in women. However, this enhanced immune response makes women more vulnerable to inflammatory and autoimmune diseases [12].

\section{Innate immunity}

Phagocytic responses Neutrophils and macrophages in females generally have a higher activation and phagocytic activity. Female dendritic cells also have higher TLR-7 and Type 1-IFN response [13]. This may result in prompt virus sensing and antiviral responses in females in the initial phase of infection.

Cytokines Notably, despite more robust immune responses in women, elevated levels of cytokines particularly IL-8 and IL-18 at the baseline, and CCL5 over the disease course were observed in men with COVID-19. This pro-inflammatory cytokine profile was strongly correlated to more severe COVID-19 outcomes, suggesting that deregulated inflammation may turn counterproductive [14]. Interestingly, these cytokine levels were also higher levels of CD14, CD16+ non-classical monocytes. Similarly, in women the production of pro-inflammatory IL-6 in viral infections is lower than in men and is correlated with better COVID-19 outcomes possibly due to reduced inflammation [15].

\section{Adaptive immunity}

T cells CD4+ and CD8+ T cells in women generate more robust responses to viral infections [16]. The Yale study reported lower $\mathrm{T}$ cell levels in males with worsening disease as compared to females. Moreover, number of activated CD8 $\mathrm{T}$ cells were significantly higher in females [14]. Higher activity of $\mathrm{T}$ cells may in turn contribute to potentially better antiviral adaptive immune response in females, which may lead to greater viral clearance.

B cells and humoral immunity Women also tend to produce higher titres of antibodies, which remain in the circulation longer, conferring long-lasting protection against COVID19 infection [14]

These primal differences in the way both sexes react to an inciting event may be ingrained in the differences in the very genetic fabric of both sexes, and their interplay with other potential contributing factors, notably sex hormones.

\section{Factors determining differences in immune response to SARS-CoV-2}

\section{Sex chromosome genes}

Immunity-related genes The possible biallelic overexpression of immune modulatory genes on the $\mathrm{X}$ chromosome in females could explain the more robust response of females to viral infection and vaccination and may also confer additional protection against COVID-19 [17].

The endosomal Toll-like receptor 7 (TLR7) is a significant factor involved in the recognition of viral antigens and mounting an effective antiviral immune response [18, 19]. Increased expression of the TLR-7 gene, which is located on the $\mathrm{X}$ chromosome, has been reported in women due to the ability of the TLR-7 gene to escape X-chromosome inactivation. Higher TLR-7-driven IFN- $\alpha$ production by 
plasmacytoid dendritic cells (pDCs) in response to acute HIV-1 infection has been reported in females as compared to males [18-20]. Rare malfunctioning variants of the TLR7 gene were found in a case series of 4 young male patients, with severe respiratory complications due to COVID19, with a negative impact on both type I and type II IFN responses [21]. Increased TLR-7 expression may lead to increased cytokine production and faster viral clearance in women. The pandemic has led to the identification of several polymorphisms in men, previously considered to be innocuous subclinical primary immunodeficiencies fairly common in the population, which may now be the very basis of the gender bias in the predisposition to life-threatening COVID$19[4,5]$.

Other genes may also be responsible for the sex-based disparity in immune response to SARS-CoV-2. These may include X-linked genes, including the ones coding for Interleukins, FOXP3, XIST, and TLR-8, which may be similarly upregulated in females due to biallelic expression, as well as Y-linked genes (SRY and SOX9), which would have greater expression in males, and thus may also possibly contribute to lower viral loads and lesser inflammation in females as compared to males [22-29]. Table 1 summarises the genes that may be responsible for the sex-based disparity in immune response as well as their function.

ACE-2 The angiotensin-converting enzyme 2 (ACE-2) receptor has a major role in the disease pathogenesis of COVID-19, acting as the receptor for viral entry of SARSCoV-2 into its target cells, primarily alveolar type 2 pneumocytes [30]. However, the ACE-2 receptor also has an important role in organ protection, as it inactivates the active forms, Angiotensin I and II by conversion into Angiotensin $1-9$, and 1-7 respectively, and thus reduces the chances of development of pulmonary oedema during COVID-19 [31, 32]. This may explain the apparent paradoxical protective effect that increased ACE-2 expression may confer against severe COVID-19 clinical outcomes in women, despite acting as the very vessel for its pathogen.

The position of the ACE-2 receptor gene on the X-chromosome, $\mathrm{Xp} 22.2$, is such that it escapes $\mathrm{X}$-inactivation in women [33]. Therefore, this leads to increased ACE-2 receptor expression in female type 2 pneumocytes, plausibly allowing the ACE- 2 receptors unbound by SARS-COV-2 Receptor binding domains, to catalytically cleave Angiotensin II and offer protection against pulmonary oedema. The absence of this biallelic expression in men may result in the lack of availability of sufficient free ACE-2 receptors to cleave Angiotensin II, and a resultant reduced protective effect.

\section{Sex hormones}

Another plausible mechanism of gender bias is the obvious difference in sex hormones between the genders, and their effect on innate and adaptive immunity. While androgens like testosterone are known to be immunosuppressive, oestrogen tends to be more versatile in its character, and may enhance or deplete immune response, based on its concentration, distribution, and expression of its receptors [34].

\section{Role of oestrogen}

Oestrogen receptors ER $\alpha$ and $\operatorname{ER} \beta$ are found on different lymphoid cells and are involved in their maturation and regulation. Oestrogen is known to play a role in monocyte/ macrophage recruitment, and regulation of cytokines and antigen presentation. Oestrogen, through its receptor signalling, has also been shown to promote pattern recognition TLR mediated production of cytokines including antiviral IFN- $\alpha$ by plasmacytoid dendritic cells [34-36].

Table 1 Genes that may influence sex-based disparity in immune response to SARS-CoV-2

\begin{tabular}{|c|c|}
\hline Gene & Function \\
\hline \multicolumn{2}{|l|}{ X-linked genes } \\
\hline ACE-2 & Receptor for SARS-CoV-2 spike (S1) protein, antioxidant, anti-inflammatory \\
\hline $\begin{array}{l}\text { Genes for interleukins e.g. IL2RG gene (encodes inter- } \\
\text { leukin receptor common gamma chain protein) }\end{array}$ & Cytokines responsible for modulating the immune response \\
\hline FOXP3, XIST & Transcription factors responsible for the functioning of regulatory T cells (Treg cells) \\
\hline TLR-8 & $\begin{array}{l}\text { Endosomal pattern recognition receptor responsible for activation of innate immunity } \\
\text { (TLR-8 binds to SARS-CoV-2 ssRNA, recruits MyD88, and activates NF- } \mathrm{kB} \text { ) }\end{array}$ \\
\hline \multicolumn{2}{|l|}{ Y-linked genes } \\
\hline TMPRSS2 & Promotes entry of SARS-CoV-2 \\
\hline SRY, SOX9 & Encodes proteins involved in the immune response to viral infections \\
\hline
\end{tabular}


Based on the similar trends of higher mortality in males during the SARS-CoV pandemic, male mouse models reported increased viral load, which correlated with higher susceptibility to critical SARS-CoV infection. Additionally, increased mortality was seen in female mice that were ovariectomised or treated with oestrogen receptor antagonists, indicating a protective role of oestrogen signalling in SARS$\mathrm{CoV}$ infection [37]. Thus, it seems plausible that similar protective role of oestrogen may exist in COVID-19 infections.

A cross sectional multicentric study on COVID-19 patients in Wuhan, China, demonstrated that premenopausal women had a milder severity and better outcome of COVID19 in contrast to men of the same age. Moreover, E2 and Anti-Müllerian hormone were found to be higher in patients with a non-severe disease. The higher E2 levels also correlated with lower IL-2, IL-6, IL-8 and TNF $\alpha$, suggesting an interplay between the hormonal and immune axis [35]. Oestrogen has also been shown to dampen the production of pro-inflammatory cytokines IL-12 from activated macrophages and IL-6, by directly altering CD16 [35]. This could indicate a possible role of oestrogen in preventing the COVID-19-associated cytokine storm syndrome.

Furthermore, oestrogens have been reported to downregulate the ACE- 2 receptor. A recent in vitro experiment demonstrated that $17 \beta$-oestradiol $\left(\mathrm{E}_{2}\right)$-medicated normal human bronchial epithelial (NHBE) cells expressed decreased ACE-2 mRNA when compared to controls, in contrast to no effect on TMPRSS2, suggesting that in females, lower levels of ACE-2 might be available for the SARS-CoV-2 virus to act on. However, the study was performed on only four experimental replicates, with NHBE cells from a single female donor, and the concentration of oestrogen used similar to the plasma levels of oestrogen in pregnancy. With current literature not reporting any significant association between pregnancy and a reduced risk of severe COVID19 outcomes, and previous studies conducted following the SARS-CoV pandemic reporting no gender-related differences in the expression of ACE-2 receptors, further studies are required to elucidate the effect of oestrogen on ACE-2 expression and correlated sex-related differences seen in immune response to COVID-19. [38-40].

\section{Role of testosterone}

Testosterone has a general immunosuppressive role, which may explain the greater susceptibility of males to a more severe disease progression of SARS-CoV-2 infection. Even though the exact mechanism by which androgens dampen the immune system is yet unclear, it has been speculated that testosterone might suppress $\mathrm{T}_{\mathrm{H}} 2$ and $\mathrm{Th} 17$ cell function and hence affect the adaptive immune response [41, 42]. Some studies have shown that testosterone might downregulate the production of cytokines such as IFN $\gamma$ and TNF $\alpha$, and increase anti-inflammatory cytokines such as IL-10 [43]. Testosterone might also play a positive regulatory role in the expression of TMPRSS2, which enhances viral entry of SARS-CoV-2 [44], suggesting that testosterone could be a possible factor leading to higher cases of COVID-19 and mortality in men as compared to women. On the contrary, a study from Italy on a cohort of 31 COVID-19 patients demonstrated that lower levels of total testosterone and calculated free testosterone at baseline correlated with worsening clinical condition, while also being significantly associated with increased neutrophils and decreased lymphocytes [45]. This implies that within men, lower levels of testosterone might increase the risk of a severe infection.

Although scientific evidence is still not conclusive, with information still evolving regarding the influence of sex hormones in COVID-19, there appears to be significant potential in oestrogen trials for improving immunity in men affected by COVID-19. Table 2 shows a list of ongoing trails for management of COVID-19.

\section{Gender-specific differences in COVID-19 manifestations}

Not only is there an increased mortality observed in men, but a disproportionately high morbidity is seen as well, with the difference in the clinical outcomes between the sexes narrowing as the age increases morbidity increased.

\section{Thrombosis in COVID-19}

The mortality observed in COVID-19 might be attributed to coagulopathies and thrombotic events. In an autopsy series, microvascular thrombosis of pulmonary vasculature was observed, and seven out of 12 patients suffered from deep vein thrombosis, while in another study venous thromboembolism was found in $69 \%$ of critically ill patients [46]. A single centre study from Italy reported thromboembolic incidents in $8 \%$ of hospitalised patients [47]. Similarly, Tang et al. reported overt-DIC in $71.4 \%$ non-survivor patients with COVID-19, while $76 \%$ of the non-survivor group were males [48]. The physiological differences between men and women may provide insights to intrinsic protective mechanisms against Thromboembolism that confer a reduced risk of COVID-19-associated coagulopathy in women, and consequently reduced mortality in women.

COVID-19-associated coagulopathy might be explained by rise in inflammatory cytokines during the disease course, increased levels of angiotensin II, increased hypercoagulability and platelet dysfunction, with age and existing co-morbidities such as hypertension being major risk factors [49].

A significant oestrogen-dependent and NO and prostacyclin-independent suppression of neutrophil recruitment and platelet adhesion on endothelial cells, via the 


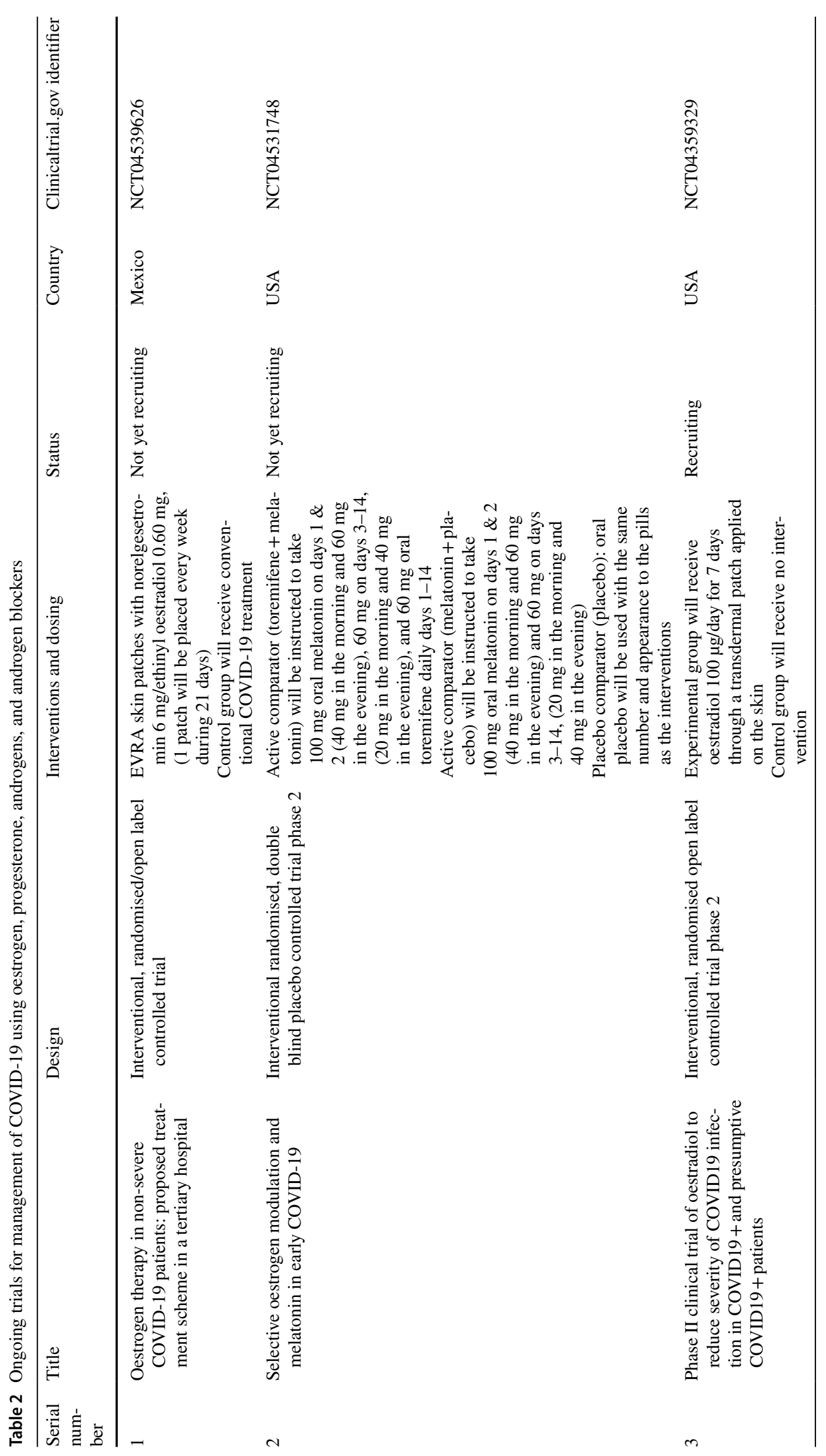




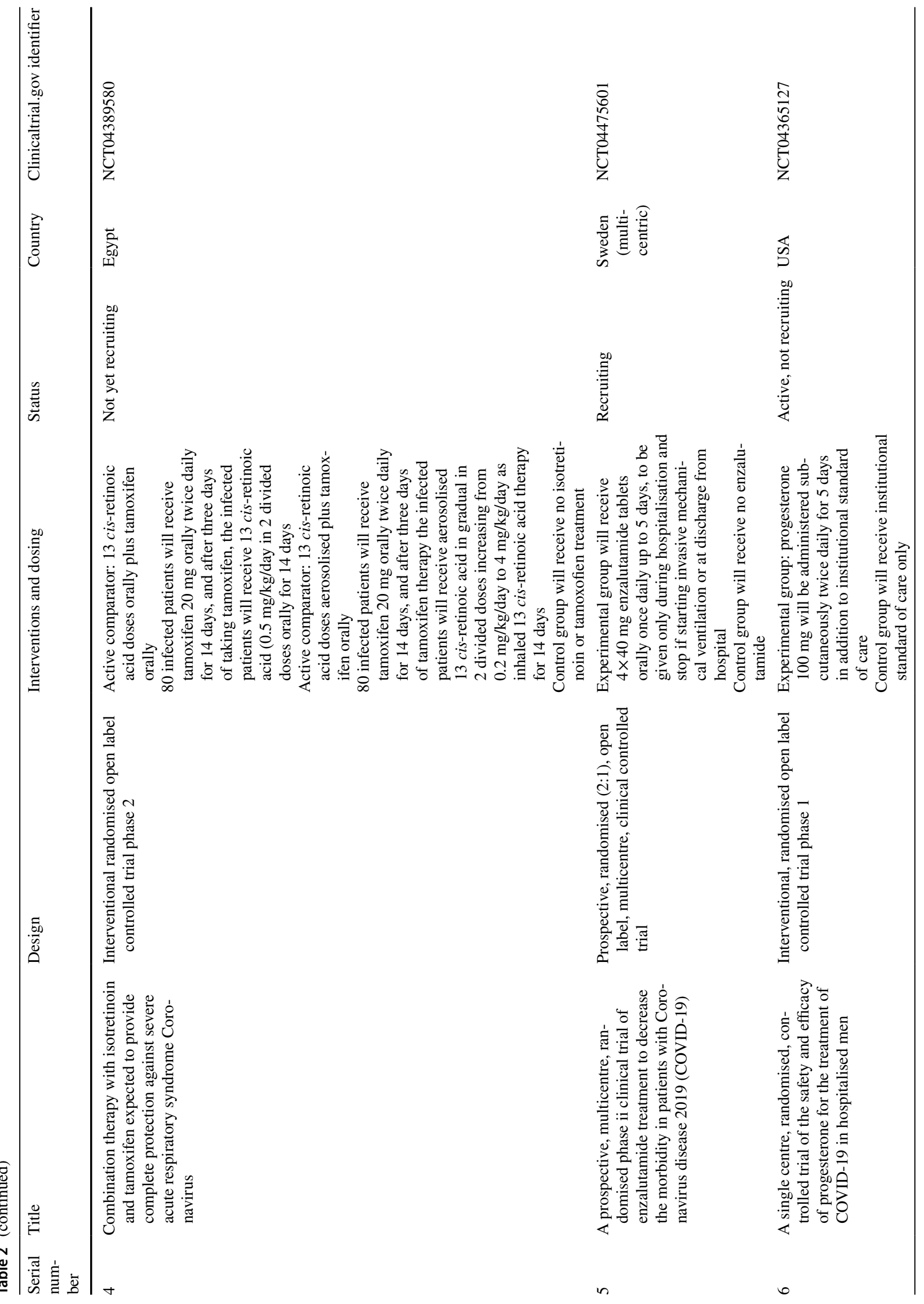




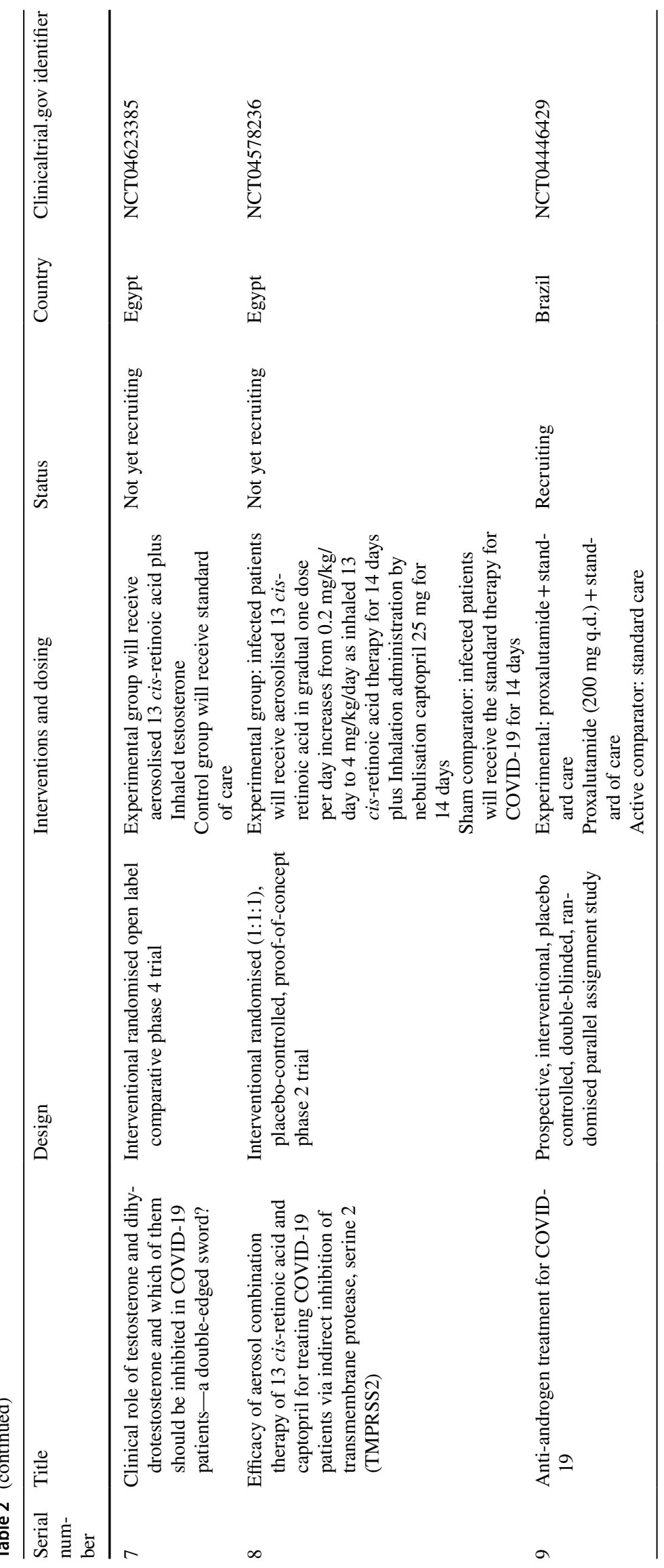


oestrogen-mediated decrease in expression of P-selectin, an adhesion molecule that facilitates leucocyte recruitment has been reported in women [50]. Furthermore, sex-based differences in platelet biology, including platelet receptor signalling, membrane composition, response to nutrients (especially polyunsaturated fatty acids and flavonoids), aggregability, platelet count, as well as the release of thromboxane A2, prostacyclin, and serotonin, which confer a protective effect against atherosclerotic plaque formation in women, may explain decreased frequency of coagulopathyassociated mortality in women.

\section{COVID-19 and autoimmunity}

The pro-thrombotic state and diffuse coagulopathy observed in several severe COVID-19 cases bares resemblance to the fatal multi-organ failure in the Antiphospholipid Syndrome (APS) due to activation of inflammatory and thrombotic pathways triggered by autoantibodies. Numerous studies have speculated a correlation of COVID-19-associated coagulopathy to antiphospholipid (aPL) antibodies found COVID-19 patients sera [51]. Previously, autoimmune responses were also found in some studies on SARS-CoV infections, and in general viral infections are known to induce transient production of aPL antibodies [52]. Zhang et al. reported $47 \%$ of 66 critical COVID-19 patients having aPLs, out of which the most common were IgA anti- $\beta_{2}$ GPI antibodies [53]. In a prior study, Zhang et al. had reported multiple infarcts and coagulopathy in 3 severely ill patients with COVID-19, who also tested positive for anti- $\beta_{2^{-}}$ glycoprotein I IgG and IgA antibodies along with anticardiolipin IgA antibodies [54]. In a cohort of 172 COVID-19 patients, Zuo et al. isolated eight variants of aPL antibodies and identified at least one type of antibody in $52 \%$ of the samples, with aCL IgM, aPS/PT IgG and aPS/PT IgM being the most commonly detected. Clinically, greater concentrations of these autoantibodies were associated with significant neutrophil activation including neutrophil extracellular traps (NETs) formation in a process called NETosis, along with increased platelet count and impaired renal function and respiratory complications. Recently, the activation of NETosis in human lymphocytes by SARS-CoV-2 was demonstrated for the first time, in a process associated with increased levels of reactive oxygen species (ROS) in neutrophils. This NET-ROS pathway may possibly be responsible for extensive microthrombus formation, one most insidious and serious complications of COVID-19. Additionally, Zuo et al. reported that $\operatorname{IgG}$ from these patients promoted venous thrombosis in mice model studies [51, 55].

These findings suggest that autoantibodies might play a role in the aetiology of COVID-19-associated coagulopathy by potentiating the already existing systemic inflammation and pro-thrombotic state and consequently lead to a severe form of the disease. It is also known that autoimmune disease are more common amongst women [56]. Further investigation is required to establish the exact basis of autoimmunity in COVID-19 and its influence on sex-based disparity of COVID-19 infections.

\section{COVID-19 and pregnancy}

Current data on COVID-19 suggests that pregnant women face a similar risk to contracting a severe disease as nonpregnant women and the general population $[57,58]$. On the contrary, in previous outbreaks of SARS and MERS, pregnant women were found to undergo significant morbidity and mortality, including poor obstetric outcomes and risk of preterm birth $[59,60]$. Generally, pregnant women are more susceptible to infections, owing to the physiological cardiopulmonary and immunological changes that occur during pregnancy. The prevalence of a Th2 system in the cell-mediated immune response during pregnancy causes a higher vulnerability to viral infections. In context of COVID-19, the already altered pulmonary function predisposes to a higher risk of developing lung consolidation and respiratory failure [61]. Furthermore, the pro-thrombotic state in pregnancy can lead to significant morbidity due to COVID-19-associated coagulopathy [62]. Although the case fatality rate in pregnant women with COVID-19 seems to be parallel to that in women of reproductive age, some cases have reported a severe COVID-19 sequalae [39]. According to the Centre for Disease Control in the United States, pregnant women required more intensive care unit admissions as compared to non-pregnant women, and often requiring mechanical ventilation [63]. Among foetal complications, preterm births was the most common, however, long-term foetal complications are yet to be seen [59].

\section{Sex-biased patterns in infectious diseases and vaccine response}

Sex-based disparities have been widely researched and reported in susceptibility and outcomes of infectious diseases and efficacy of vaccines [15, 64]. During the SARS outbreak in 2003, case fatality rates were higher in men than in females, and similar trends were seen in the MERS outbreak as well [6]. In HIV infections, studies have shown females to have higher CD4+ counts and stronger activation of CD8+ T cells, coupled with lower viral loads, relating to enhanced innate viral sensing and greater IFN $\alpha$ production [20]. Similarly, in Hepatitis B infection, males have been reported to have higher HBV DNA titres, and an increased susceptibility to developing hepatocellular carcinoma, which has been positively correlated to testosterone concentration and certain androgen receptor genes [65, 66]. Furthermore, 
in both adults and children, females were found to generate an increased anti-HBV antibody titre as compared to males [16]. In contrast, while exposure rates to Influenza A viruses are greater in men, higher fatality is reported in younger women [67]. However, females mount a stronger antibody response to the Influenza A vaccine, possibly regulated by greater TLR7 expression and induction of B cells by oestradiol [64]. Hence, several studies have concluded that females tend to produce a more efficacious response to vaccines, although time shall answer this question regarding COVID-19.

\section{Behavioural risk factors and social constructs}

Gendered differences in behavioural risk factors may also be a contributing factor in the apparent differences in mortality between men and women. Smoking has been linked to higher disease severity in COVID-19 and has also been associated with higher expression of ACE-2. Since smoking is more prevalent in men it can predispose men to a worse clinical prognosis [68]. Studies have also shown that women tend to use more healthcare services than men and employ better hygiene practices [69]. A survey from the United States in the at the onset of the pandemic reported that adult men were less determined to observe behavioural changes such as social distancing and wearing masks, and exhibited lesser COVID-19 worry and concern [70]. This may contribute to greater susceptibility to COVID-19 infection in men.

In many parts of the world, including ours, men have a greater proclivity to work outside the home, travel via public transport, and participate in large gatherings such as congregational religious ceremonies as compared to women, inevitably predisposing them to a higher risk of infection due to the greater risk of exposure. However, social constructs and norms, that lead to a disparity in autonomy over healthcare rights and access to healthcare services between the sexes, especially in lower socioeconomic settings, with women often having to bear with delayed hospital visits and inadequate treatment, also merit exploration.
Gender specific behavioural differences seem to only contribute a minor role to COVID-19 sex bias. Physiologically, the interplay between sex hormones and immune effectors primarily drives the drift between sexes in regard to infectious diseases. Guerra-Silveira et al. reported sex-related behaviour differences that lead to differences in exposureprevalence of diseases, to be significant only in Schistosomiasis and Leptospirosis, while the in the vast majority of diseases, the disparity in incidence and severity of disease was inextricably linked to physiological differences between the sexes. Even though this study primarily investigated the epidemiology of bacterial and parasitic infections such as Tuberculosis and Dengue, the models for disease epidemiology in these high prevalence diseases, may be extrapolated to explain the sex based differences in incidence and clinical outcomes in COVID-19, which is also a high burden disease [71]. Figure 1 summarises the factors influencing sex-based disparity observed in COVID-19.

\section{Conclusion and implications for the future}

Global data strongly indicates that a sex-based disparity exists in COVID-19 clinical outcomes, with men being more affected by initial SARS-CoV-2 infection, hospitalisation and poor clinical outcomes. This sex-based disparity similarly exists in the immune response to infections and inflammatory diseases.

Several factors and mechanisms may explain this disparity, including intrinsic differences in innate and adaptive immunity, plausibly arising from genetic differences in the expression of immune regulatory genes between men and women, the role of crosstalk between sex hormones, as well as differences in gender specific behaviours. These differences confer a protective advantage to women against COVID-19, with women consistently reported to have lower viral loads, lesser inflammation, better clinical outcomes and lesser mortality as compared to men.

These differences, particularly the immunological divergence in response to viral infection, could potentially impact COVID-19 disease course, and antiviral drugs and vaccines. Furthermore, these immunological factors, which confer protection against COVID-19, may be exploited to develop therapeutic strategies to improve clinical outcomes.

Author contributions HR and LG conceptualisation. HR, PS, and LG data curation. HR, PS, LG, and OB formal analysis. HR, PS, LG, and OB visualisation. HR and LG writing-original draft. PS writing review and editing.

Funding None. 
Fig. 1 Factors contributing to severity of COVID-19 outcomes and mortality in males

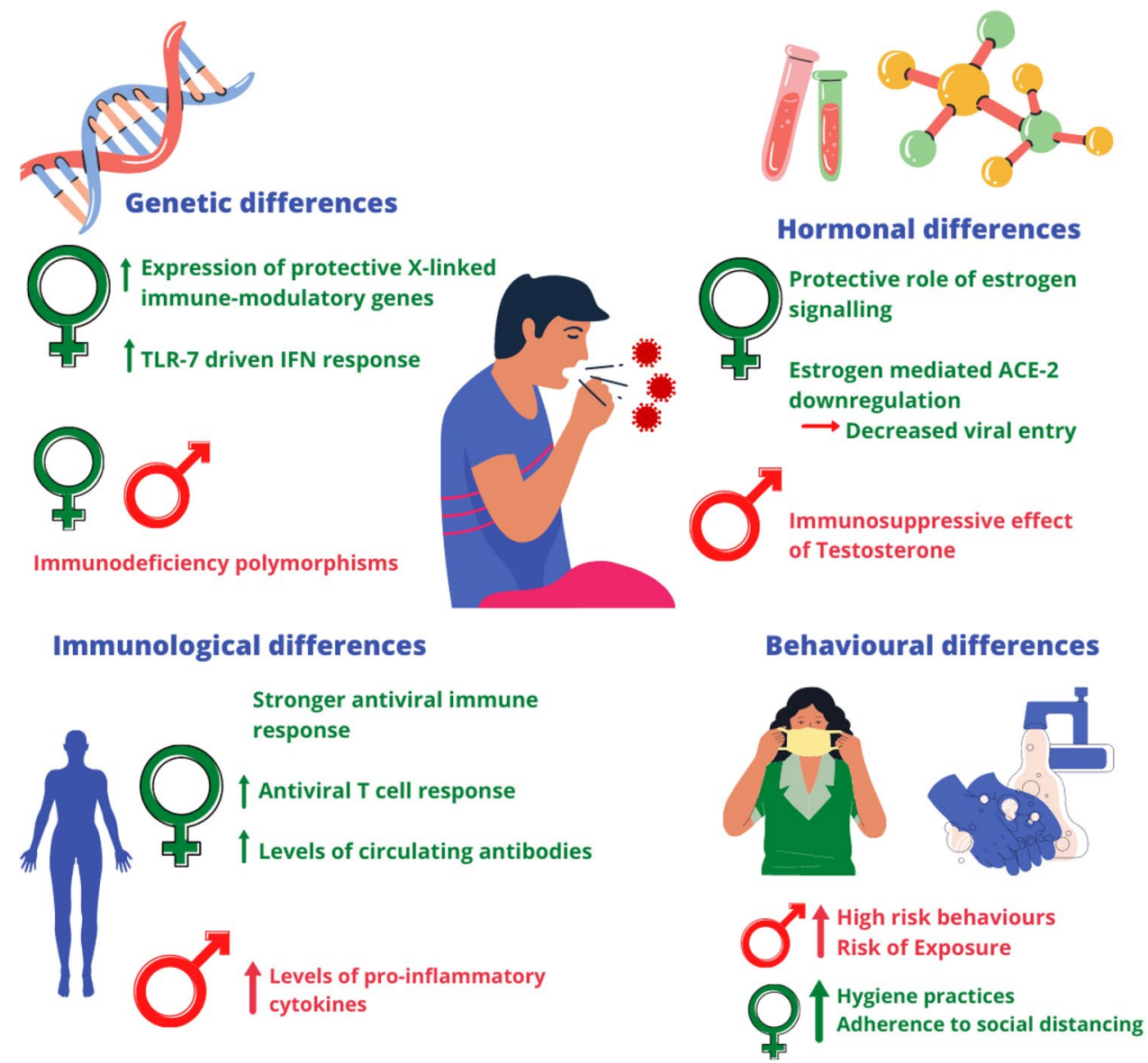

\section{Declarations}

Conflict of interest The authors declare that there is no conflict of interest.

\section{References}

1. Chen J, Qi T, Liu L et al (2020) Clinical progression of patients with COVID-19 in Shanghai, China. J Infect 80:e1-e6. https://doi. org/10.1016/j.jinf.2020.03.004

2. Xie J, Tong Z, Guan X et al (2020) Clinical characteristics of patients who died of Coronavirus disease 2019 in China. JAMA Netw Open. https://doi.org/10.1001/jamanetworkopen.2020.5619

3. Epidemiology Working Group for NCIP Epidemic Response, Chinese Center for Disease Control and Prevention (2020) The epidemiological characteristics of an outbreak of 2019 novel coronavirus diseases (COVID-19) in China. Zhonghua Liu Xing Bing Xue Za Zhi 41:145-151. https://doi.org/10.3760/cma.j.issn. 0254-6450.2020.02.003

4. Scully EP, Haverfield J, Ursin RL et al (2020) Considering how biological sex impacts immune responses and COVID-19 outcomes. Nat Rev Immunol 20:442-447. https://doi.org/10.1038/ s41577-020-0348-8
5. The sex, gender and COVID-19 project. Global health 50/50. https://globalhealth5050.org/the-sex-gender-and-covid-19-proje ct/. Accessed 07 Apr 2021

6. Karlberg J, Chong DSY, Lai WYY (2004) Do men have a higher case fatality rate of severe acute respiratory syndrome than women do? Am J Epidemiol 159:229-231. https://doi.org/10. 1093/aje/kwh056

7. Alghamdi IG, Hussain II, Almalki SS et al (2014) The pattern of Middle East respiratory syndrome coronavirus in Saudi Arabia: a descriptive epidemiological analysis of data from the Saudi Ministry of Health. Int J Gen Med 7:417-423. https://doi.org/ 10.2147/IJGM.S67061

8. Eular_Covid_19_Registry_Report_20210302.pdf

9. Hasseli R, Mueller-Ladner U, Hoyer BF et al (2021) Older age, comorbidity, glucocorticoid use and disease activity are risk factors for COVID-19 hospitalisation in patients with inflammatory rheumatic and musculoskeletal diseases. RMD Open. https://doi.org/10.1136/rmdopen-2020-001464

10. Sharma G, Volgman AS, Michos ED (2020) Sex differences in mortality from COVID-19 pandemic. JACC Case Rep 2:147140. https://doi.org/10.1016/j.jaccas.2020.04.027

11. Gasparyan AY, Ayvazyan L, Blackmore H, Kitas GD (2011) Writing a narrative biomedical review: considerations for authors, peer reviewers, and editors. Rheumatol Int 31:14091417. https://doi.org/10.1007/s00296-011-1999-3 
12. Klein SL, Flanagan KL (2016) Sex differences in immune responses. Nat Rev Immunol 16:626-638. https://doi.org/10.1038/ nri.2016.90

13. Conti P, Younes A (2020) Coronavirus COV-19/SARS-CoV-2 affects women less than men: clinical response to viral infection. J Biol Regul Homeost Agents 34:339-343. https://doi.org/10. 23812/Editorial-Conti-3

14. Yale IMPACT Research Team, Takahashi T, Ellingson MK et al (2020) Sex differences in immune responses that underlie COVID-19 disease outcomes. Nature 588:315-320. https://doi. org/10.1038/s41586-020-2700-3

15. Fischinger S, Boudreau CM, Butler AL et al (2019) Sex differences in vaccine-induced humoral immunity. Semin Immunopathol 41:239-249. https://doi.org/10.1007/s00281-018-0726-5

16. Klein SL (2012) Sex influences immune responses to viruses, and efficacy of prophylaxis and therapeutic treatments for viral diseases. BioEssays 34:1050-1059. https://doi.org/10.1002/bies. 201200099

17. Schurz H, Salie M, Tromp G et al (2019) The X chromosome and sex-specific effects in infectious disease susceptibility. Hum Genom 13:2. https://doi.org/10.1186/s40246-018-0185-z

18. Souyris M, Cenac C, Azar P et al (2018) TLR7 escapes X chromosome inactivation in immune cells. Sci Immunol. https://doi.org/ 10.1126/sciimmunol.aap8855

19. Berghöfer B, Frommer T, Haley G et al (2006) TLR7 ligands induce higher IFN-alpha production in females. J Immunol 177:2088-2096. https://doi.org/10.4049/jimmunol.177.4.2088

20. Meier A, Chang JJ, Chan ES et al (2009) Sex differences in the toll-like receptor-mediated response of plasmacytoid dendritic cells to HIV-1. Nat Med 15:955-959. https://doi.org/10.1038/nm. 2004

21. van der Made CI, Simons A, Schuurs-Hoeijmakers J et al (2020) Presence of genetic variants among young men with severe COVID-19. JAMA 324:663. https://doi.org/10.1001/jama.2020. 13719

22. Pagliaro P, Penna C (2005) Rethinking the renin-angiotensin system and its role in cardiovascular regulation. Cardiovasc Drugs Ther 19:77-87. https://doi.org/10.1007/s10557-005-6900-8

23. Cheng Z, Zhou J, To KK-W et al (2015) Identification of TMPRSS2 as a susceptibility gene for severe 2009 pandemic $\mathrm{A}(\mathrm{H} 1 \mathrm{~N} 1)$ influenza and $\mathrm{A}(\mathrm{H} 7 \mathrm{~N} 9)$ influenza. J Infect Dis 212:1214-1221. https://doi.org/10.1093/infdis/jiv246

24. Ghosh S, Klein RS (2017) Sex drives dimorphic immune responses to viral infections. J Immunol 198:1782-1790. https:// doi.org/10.4049/jimmunol.1601166

25. Márquez EJ, Trowbridge J, Kuchel GA et al (2020) The lethal sex gap: COVID-19. Immun Ageing 17:13. https://doi.org/10.1186/ s12979-020-00183-z

26. Tomlins SA, Rhodes DR, Perner S et al (2005) Recurrent fusion of TMPRSS2 and ETS transcription factor genes in prostate cancer. Science 310:644-648. https://doi.org/10.1126/science.1117679

27. Setlur SR, Mertz KD, Hoshida Y et al (2008) Estrogen-dependent signaling in a molecularly distinct subclass of aggressive prostate cancer. J Natl Cancer Inst 100:815-825. https://doi.org/10.1093/ jnci/djn 150

28. Bukowska A, Spiller L, Wolke C et al (2017) Protective regulation of the ACE2/ACE gene expression by estrogen in human atrial tissue from elderly men. Exp Biol Med (Maywood) 242:1412-1423. https://doi.org/10.1177/1535370217718808

29. Fagone P, Ciurleo R, Lombardo SD et al (2020) Transcriptional landscape of SARS-CoV-2 infection dismantles pathogenic pathways activated by the virus, proposes unique sex-specific differences and predicts tailored therapeutic strategies. Autoimmun Rev 19:102571. https://doi.org/10.1016/j.autrev.2020.102571
30. Hoffmann M, Kleine-Weber H, Schroeder S et al (2020) SARSCoV-2 cell entry depends on ACE2 and TMPRSS2 and is blocked by a clinically proven protease inhibitor. Cell 181:271-280.e8. https://doi.org/10.1016/j.cell.2020.02.052

31. Zhang H, Penninger JM, Li Y et al (2020) Angiotensin-converting enzyme 2 (ACE2) as a SARS-CoV-2 receptor: molecular mechanisms and potential therapeutic target. Intensive Care Med 46:586-590. https://doi.org/10.1007/s00134-020-05985-9

32. Kuba K, Imai Y, Rao S et al (2005) A crucial role of angiotensin converting enzyme 2 (ACE2) in SARS coronavirus-induced lung injury. Nat Med 11:875-879. https://doi.org/10.1038/nm1267

33. Carrel L, Willard HF (2005) X-inactivation profile reveals extensive variability in X-linked gene expression in females. Nature 434:400-404. https://doi.org/10.1038/nature03479

34. Khan D, Ansar Ahmed S (2016) The immune system is a natural target for estrogen action: opposing effects of estrogen in two prototypical autoimmune diseases. Front Immunol. https://doi.org/10. 3389/fimmu.2015.00635

35. Ding T, Zhang J, Wang T et al (2020) Potential influence of menstrual status and sex hormones on female severe acute respiratory syndrome Coronavirus 2 infection: a cross sectional multicenter study in Wuhan, China. Clin Infect Dis. https://doi.org/10.1093/ cid/ciaa1022

36. Seillet $\mathrm{C}$, Laffont $\mathrm{S}$, Trémollières $\mathrm{F}$ et al (2012) The TLR-mediated response of plasmacytoid dendritic cells is positively regulated by estradiol in vivo through cell-intrinsic estrogen receptor $\alpha$ signaling. Blood 119:454-464. https://doi.org/10.1182/ blood-2011-08-371831

37. Channappanavar R, Fett C, Mack M et al (2017) Sex-based differences in susceptibility to severe acute respiratory syndrome coronavirus infection. J Immunol 198:4046-4053. https://doi.org/ 10.4049/jimmunol.1601896

38. Xudong X, Junzhu C, Xingxiang W et al (2006) Age- and gender-related difference of ACE2 expression in rat lung. Life Sci 78:2166-2171. https://doi.org/10.1016/j.lfs.2005.09.038

39. Stelzig KE, Canepa-Escaro F, Schiliro M et al (2020) Estrogen regulates the expression of SARS-CoV-2 receptor ACE2 in differentiated airway epithelial cells. Am J Physiol Lung Cell Mol Physiol 318:L1280-L1281. https://doi.org/10.1152/ajplung. 00153.2020

40. Liu J, Ji H, Zheng $\mathrm{W}$ et al (2010) Sex differences in renal angiotensin converting enzyme 2 (ACE2) activity are $17 \beta$-oestradioldependent and sex chromosome-independent. Biol Sex Dif 1:6. https://doi.org/10.1186/2042-6410-1-6

41. Roved J, Westerdahl H, Hasselquist D (2017) Sex differences in immune responses: hormonal effects, antagonistic selection, and evolutionary consequences. Horm Behav 88:95-105. https://doi. org/10.1016/j.yhbeh.2016.11.017

42. Trigunaite A, Dimo J, Jørgensen TN (2015) Suppressive effects of androgens on the immune system. Cell Immunol 294:87-94. https://doi.org/10.1016/j.cellimm.2015.02.004

43. Kadel S, Kovats S (2018) Sex hormones regulate innate immune cells and promote sex differences in respiratory virus infection. Front Immunol 9:1653. https://doi.org/10.3389/fimmu.2018. 01653

44. Pozzilli P, Lenzi A (2020) Commentary: testosterone, a key hormone in the context of COVID-19 pandemic. Metabolism 108:154252. https://doi.org/10.1016/j.metabol.2020.154252

45. Rastrelli G, Di Stasi V, Inglese F et al (2020) Low testosterone levels predict clinical adverse outcomes in SARS-CoV-2 pneumonia patients. Andrology. https://doi.org/10.1111/andr.12821

46. Wichmann D, Sperhake J-P, Lütgehetmann M et al (2020) Autopsy findings and venous thromboembolism in patients with COVID-19. Ann Intern Med. https://doi.org/10.7326/M20-2003 
47. Lodigiani C, Iapichino G, Carenzo L et al (2020) Venous and arterial thromboembolic complications in COVID-19 patients admitted to an academic hospital in Milan, Italy. Thromb Res 191:9-14. https://doi.org/10.1016/j.thromres.2020.04.024

48. Tang N, Li D, Wang X, Sun Z (2020) Abnormal coagulation parameters are associated with poor prognosis in patients with novel coronavirus pneumonia. J Thromb Haemost 18:844-847. https://doi.org/10.1111/jth.14768

49. Miesbach W, Makris M (2020) COVID-19: coagulopathy, risk of thrombosis, and the rationale for anticoagulation. Clin Appl Thromb Hemost. https://doi.org/10.1177/1076029620938149

50. Villar IC, Scotland RS, Khambata RS et al (2011) Suppression of endothelial P-selectin expression contributes to reduced cell trafficking in females: an effect independent of $\mathrm{NO}$ and prostacyclin. Arterioscler Thromb Vasc Biol 31:1075-1083. https://doi.org/10. 1161/ATVBAHA.111.223545

51. Zuo Y, Estes SK, Ali RA et al (2020) Prothrombotic autoantibodies in serum from patients hospitalized with COVID-19. Sci Transl Med 12:eabd3876. https://doi.org/10.1126/scitranslmed. abd3876

52. Xiao M, Zhang Y, Zhang S et al (2020) Antiphospholipid antibodies in critically ill patients with COVID-19. Arthritis Rheumatol 72:1998-2004. https://doi.org/10.1002/art.41425

53. Zhou Y, Han T, Chen J et al (2020) Clinical and autoimmune characteristics of severe and critical cases of COVID-19. Clin Transl Sci 13:1077-1086. https://doi.org/10.1111/cts.12805

54. Zhang Y, Xiao M, Zhang S et al (2020) Coagulopathy and antiphospholipid antibodies in patients with Covid-19. N Engl J Med 382:e38. https://doi.org/10.1056/NEJMc2007575

55. Arcanjo A, Logullo J, Menezes CCB et al (2020) The emerging role of neutrophil extracellular traps in severe acute respiratory syndrome Coronavirus 2 (COVID-19). Sci Rep 10:19630. https:// doi.org/10.1038/s41598-020-76781-0

56. Angum F, Khan T, Kaler J et al (2020) The prevalence of autoimmune disorders in women: a narrative review. Cureus. https://doi. org/10.7759/cureus.8094

57. Knight M, Bunch K, Vousden N et al (2020) Characteristics and outcomes of pregnant women admitted to hospital with confirmed SARS-CoV-2 infection in UK: national population based cohort study. BMJ 369:m2107. https://doi.org/10.1136/bmj.m2107

58. Vlachodimitropoulou Koumoutsea E, Vivanti AJ, Shehata N et al (2020) COVID-19 and acute coagulopathy in pregnancy. J Thromb Haemost 18:1648-1652. https://doi.org/10.1111/jth. 14856

59. Khalil A, Kalafat E, Benlioglu C et al (2020) SARS-CoV-2 infection in pregnancy: a systematic review and meta-analysis of clinical features and pregnancy outcomes. EClinicalMedicine 25:100446. https://doi.org/10.1016/j.eclinm.2020.100446
60. Di Mascio D, Khalil A, Saccone G et al (2020) Outcome of coronavirus spectrum infections (SARS, MERS, COVID-19) during pregnancy: a systematic review and meta-analysis. Am J Obstet Gynecol MFM 2:100107. https://doi.org/10.1016/j.ajogmf.2020. 100107

61. Dashraath P, Wong JLJ, Lim MXK et al (2020) Coronavirus disease 2019 (COVID-19) pandemic and pregnancy. Am J Obstet Gynecol 222:521-531. https://doi.org/10.1016/j.ajog.2020.03.021

62. Iba T, Levy JH, Levi M et al (2020) Coagulopathy of Coronavirus disease 2019. Crit Care Med. https://doi.org/10.1097/CCM.00000 00000004458

63. Ellington S (2020) Characteristics of women of reproductive age with laboratory-confirmed SARS-CoV-2 infection by pregnancy status-United States, January 22-June 7, 2020. Morb Mortal Wkly Rep. https://doi.org/10.15585/mmwr.mm6925a1

64. Fink AL, Engle K, Ursin RL et al (2018) Biological sex affects vaccine efficacy and protection against influenza in mice. Proc Natl Acad Sci USA 115:12477-12482. https://doi.org/10.1073/ pnas. 1805268115

65. Wang S-H, Yeh S-H, Lin W-H et al (2009) Identification of androgen response elements in the enhancer I of hepatitis B virus: a mechanism for sex disparity in chronic hepatitis B. Hepatology 50:1392-1402. https://doi.org/10.1002/hep.23163

66. Yu M-W (2000) Androgen-receptor gene CAG repeats, plasma testosterone levels, and risk of hepatitis B-related hepatocellular carcinoma. J Natl Cancer Inst 92:2023-2028. https://doi.org/10. 1093/jnci/92.24.2023

67. WHO. Sex, gender and influenza. https://www.who.int/genderequity-rights/knowledge/9789241500111/en/. Accessed 07 Apr 2021

68. Vardavas CI, Nikitara K (2020) COVID-19 and smoking: a systematic review of the evidence. Tob Induc Dis. https://doi.org/10. 18332/tid/119324

69. Bertakis KD, Azari R, Helms LJ et al (2000) Gender differences in the utilization of health care services. J Fam Pract 49:147-152

70. Barber SJ, Kim H (2021) COVID-19 worries and behavior changes in older and younger men and women. J Gerontol B Psychol Sci Soc Sci 76:e17-e23. https://doi.org/10.1093/geronb/ gbaa068

71. Guerra-Silveira F, Abad-Franch F (2013) Sex bias in infectious disease epidemiology: patterns and processes. PLoS ONE. https:// doi.org/10.1371/journal.pone.0062390

Publisher's Note Springer Nature remains neutral with regard to jurisdictional claims in published maps and institutional affiliations. 